\title{
FisicaTIC, plataforma hardware-software para aplicaciones en física e ingeniería
}

\author{
FisicaTIC, hardware-software platform for applications in physics and engineering
}

\author{
J. E. Chaparro-Mesa ; N. Barrera-Lombana iD ; F. A. León-Socha iD
}

\begin{abstract}
One of the main problems facing the teaching methods of basic sciences and engineering is the separation of theoretical knowledge from practical training. In this sense, laboratory experiences become very useful teaching strategies; however, implementing them requires physical infrastructure, equipment and materials that originate large economic investments. In response to this problem, FisicaTIC arises, a hardware-software platform that allows various physics and engineering practices related to the kinematics and dynamics of bodies. This proposal aims to develop learning skills, with the help of the student's own technological tools, (Smartphone, PC or Tablet). For the software development, "SCRUM" was used as a methodological basis, however, this methodology was integrated into a $\mathrm{V}$ model, taking into account that this development technique is efficient for the materialization of technological products that require the implementation of hardware and software embedded subsystems, as it allows to specify in greater detail the tools to be used in each of the product development phases. For the validation and verification of the software and hardware built, the IEEE 1012-2016 standard was used as a reference. Finally, it should be mentioned that the results obtained through the use of FisicaTIC, allow to infer that students develop cognitive skills during their interaction with the built system, favoring the approach and understanding of concepts related to kinematics, gravitational forces and movement simple harmonic.
\end{abstract}

Index Terms - Accelerometer, Computer teaching, Data acquisition, Simple Harmonic Movement, Virtual Physics Laboratory.

Resumen- Uno de los principales problemas que enfrentan los métodos de enseñanza de las ciencias básicas y las ingenierías, es la separación del conocimiento teórico de la formación práctica. En este sentido, las experiencias en laboratorio se convierten en estrategias didácticas pedagógicas muy provechosas; sin embargo, implementarlas requiere de infraestructura física, equipos y materiales que originan grandes inversiones económicas. Como respuesta a esta problemática surge FisicaTIC, una plataforma hardware-software, que permite realizar diversas prácticas de física e ingeniería relacionadas con la cinemática y dinámica de los

Este manuscrito fue enviado el 20 de enero de 2019 y aceptado el 25 de junio de 2019. Este articulo hace parte de los productos del proyecto de investigación, FisicaTIC, financiado por la Dirección de Investigaciones de la Universidad Internacional del Trópico Americano, Unitrópico, en el año 2016.

J. E. Chaparro-Mesa. Docente Investigador. Coordinador de producción de Investigaciones, Fundación Universitaria Internacional del Trópico Americano, Unitrópico. Carrera 19 No 39-40 Yopal- Casanare Colombia. (e-mail: jorgechaparro@unitropico.edu.co) cuerpos. Esta propuesta pretende desarrollar habilidades de aprendizaje, con la ayuda de las propias herramientas tecnológicas del estudiante, (Smartphone, PC o Tablet). Para el desarrollo de software, se empleó como base metodológica "SCRUM", sin embargo, esta metodología se integró a un modelo en $\mathrm{V}$, teniendo en cuenta que esta técnica de desarrollo es eficiente para la materialización de productos tecnológicos que requieren de la implementación de subsistemas embebidos de hardware y software, ya que permite precisar con mayor nivel de detalle las herramientas a utilizar en cada una de las fases de desarrollo del producto. Para la validación y verificación del software y hardware construido. Se empleó como referente el estándar IEEE 1012-2016. Finalmente, se debe mencionar que los resultados obtenidos mediante el uso de FisicaTIC, permiten inferir que los estudiantes desarrollan habilidades cognoscitivas durante su interacción con el sistema construido, favoreciendo el abordaje y la comprensión de conceptos relacionados con la cinemática, las fuerzas gravitatorias y el movimiento armónico simple.

Palabras claves-Acelerómetro, Adquisición de datos, Enseñanza mediada por ordenador, Laboratorio Virtual de Física, Movimiento Armónico Simple.

\section{INTRODUCTION}

$\mathrm{U}$ NO de los principales problemas que enfrentan los métodos de enseñanza de las ciencias básicas y de las ingenierías, es la separación del conocimiento teórico de la formación práctica [1], [2]. En este sentido resulta trascendental que los docentes empleen diversas estrategias didácticas que les permita generar en los estudiantes la comprensión de los fenómenos que los rodean, para que así desarrollen y fortalezcan los conocimientos conceptuales, procedimentales y las competencias científicas necesarias [3]. Dentro de las estrategias didácticas pedagógicas más provechosas están las prácticas de laboratorio, las cuales generan una oportunidad valiosa en el desarrollo cognitivo y motivacional de los estudiantes [4]; sin embargo,

N. Barrera-Lombana. Director del programa de Ingeniería Electrónica de la Universidad Pedagógica y Tecnológica de Colombia, seccional Sogamoso U.P.T.C 2019. Calle 4 sur No. 15-134 Sogamoso - Boyacá Colombia. (e-mail: Nelson.barrera@uptc.edu.co)

F. A. León-Socha. Investigador, Centro de Desarrollo Tecnológico e Innovación en TIC, NetworkTIC. Calle 33 No 28-47 Yopal Casanare Colombia (e-mail: gerencia@networktic.com) 
implementarlas requieren de infraestructura física, equipos y materiales que generan grandes inversiones económicas.

Por otra parte, se encuentran las herramientas digitales diseñadas con fines educativos; dentro de las cuales se destacan los laboratorios virtuales por su impacto visual y sus características de animación, que simulan el ambiente de un laboratorio real, sumado a que brindan una gran variedad metodológica, flexibilidad en horarios, entornos gráficos atractivos y una notable disminución en los costos de operación [5].

Actualmente los laboratorios virtuales incorporan software, hardware y sistemas modernos de comunicación, que facilitan el acceso remoto y posibilitan el diseño y la creación de objetos mucho más reales, permitiéndole a los estudiantes mayor autonomía y participación en las pruebas, a fin de construir su propio conocimiento científico y desarrollar mejor sus competencias con ayuda de sus propias herramientas tecnológicas como el Celular, PC o Tablet [6].

En este contexto surge FisicaTIC, cuyo fin es permitir que los alumnos de física e ingenierías, despierten su interés y fortalezcan sus conocimientos, haciendo uso de sus habilidades naturales en el empleo de la tecnología. FisicaTIC, de forma general, es un laboratorio virtual de física que, mediante la integración, hardware - software - comunicación, permite que los estudiantes realicen diversas prácticas relacionadas con el movimiento armónico simple y las fuerzas gravitacionales. El dispositivo está conformado básicamente por tres subsistemas; aplicación para usuario final, hardware-comunicación, y sistema físico monitoreado y evaluado.

En cuanto al hardware consiste básicamente en un acelerómetro conectado a un microcontrolador que se encarga de leer y procesar datos relacionados con la aceleración de un objeto en un espacio tridimensional, una etapa de comunicación Bluetooth y USB-OTG a través de la cual el módulo se enlaza con dispositivos móviles como Tablet, Celulares o PC, los cuales reciben los datos en tiempo real, los procesa y grafica de acuerdo a los laboratorios planeados de forma previa.

Para la materialización del hardware se utilizaron criterios de diseño tales como, desempeño, disponibilidad de componentes en el mercado, uso de elementos de calidad comercial (Comercial-Off-The-Shelf. COTS), facilidad de implementación, robustez y costos de implementación.

En cuanto al software se utilizó como base metodológica Scrum [7], la cual hace parte de las Metodologías Agiles y como herramienta de programación se trabajó con Basic4Android [8]. La codificación por su parte se realizó teniendo en cuenta cinco módulos definidos; Intro, Menú de Temas, Menú de Subtemas y Menú de Opciones de Laboratorio. En este último se encuentra la guía del laboratorio, el panel de control principal, el formulario del informe. Por su parte en el panel de control se encuentran las opciones para realizar la conexión con el módulo FisicaTIC, registrar datos en archivo plano(CVS), capturar la pantalla (evidencias para el informe final) y habilitar la cámara del dispositivo para tomar evidencias del desarrollo del laboratorio. Así mismo con los datos almacenados en el archivo plano, se realizan los cálculos mediante ecuaciones que permiten comprobar los resultados del laboratorio desarrollado.

\section{ESTADO DEL ARTE}

Existen diversas propuestas de laboratorios virtuales con fines educativos para la enseñanza de la física, dentro de las cuales se puede citar a PhysicsSensor, esta es una plataforma hardware-software, desarrollada para realizar laboratorios básicos de enseñanza de las ciencias exactas y naturales, la cual cuenta con las siguientes aplicaciones: acelerómetro (inercial y no inercial), inclinómetro, termómetro (temperatura ambiental), barómetro, higrómetro, altímetro, luxómetro, gaussímetro, sonómetro, sonoscopio, generador de señales, espectrómetro, video tracker, regresión lineal, regresión cuadrática [9]. Xplorer $\boldsymbol{G L} \boldsymbol{X}$, es un equipo de adquisición de datos, gráficos y análisis diseñado para estudiantes y educadores de ciencias. También funciona como interfaz del sensor PASPORT cuando está conectado a un ordenador de sobremesa o portátil con el software DataStudio [10].

Por otra parte, existen laboratorios virtuales remotos que permiten realizar diferentes prácticas, como el caso de $\boldsymbol{e}$ Laboratory Project, el cual cuenta con un portal elaborado donde cada experimento remoto está acompañado de una serie de apoyos: motivación, antecedentes físicos, una guía del experimento, descripción de arreglo experimental y el acceso a la experiencia remota. Este laboratorio se encuentra alojado en la Prague Caroline University de la república Checa; dentro de las características tecnológicas, permite el acceso y registro, además proporciona video de la experiencia en directo [11], [12]. Otro laboratorio remoto es RemLabNet, Remote Laboratory Management System (RLMS), este permite gestionar laboratorios ya existentes en instituciones de Europa; dentro de las practicas propuestas por este sistema están: Péndulo simple; Caída libre I y II; Principio de Arquímedes; Plano inclinado; Experimento de Joule; Circuitos RLC; Energía en RLC; Circuitos RLC en fase; Emisión lumínica mediante de LED; Efecto Fotovoltaico; Ley de Faraday [13]. WebLabDeusto, también es un LR, el cual ha sido desarrollado y publicado como un software de código abierto, ofrece guías de usuario, manuales técnicos, descripción de cada experimento, y videos que muestran la ejecución de las experiencias [14]. Physilab es una propuesta metodológica orientada hacia la enseñanza y comprensión de la física, basada en las TIC. En este proyecto se elaboró un libro el cual contiene conceptos y ejercicios, prácticas convencionales, laboratorio virtual y prácticas de acceso remoto, lo que resulta un complemento didáctico muy interesante en la enseñanza de la física [15]. PhET, es una plataforma de acceso libre, la cual es proporcionada por la universidad de Colorado de forma gratuita, cuenta con varias pruebas de laboratorio de física, biología, matemáticas y ciencias de la tierra entre otras [16], [17].

De otra parte, en la Universidad de Vigo España desarrollaron un laboratorio virtual de código abierto para la ecuación de Schrödinger, esta plataforma consiste en un software de código abierto basado en Python para la simulación numérica de la ecuación de Schrödinger lineal y no lineal en una y dos dimensiones [18]. En esta misma línea en la Universidad Tecnológica Nacional de Argentina, construyeron un volante Inercial usando un hardware, conformado por el volante, una pesa, una cuerda inextensible y un sensor de rotación, el cual 
adquiere los datos y los transmite en tiempo real a una computadora que contiene una aplicación para tal efecto [19].

Referente a las ventajas que ofrecen los laboratorios virtuales se han realizado diversas investigaciones, dentro de las que se pueden mencionar el trabajo realizado por C. Infante Jiménez, en el que se analizan las ventajas y desventajas de la utilización de los laboratorios virtuales como actividad complementaria en las asignaturas teórico-prácticas, y proponiendo un entorno blended learning (b-learning), que es una mezcla de actividades presenciales y virtuales [20]. Por otra parte en Universitas Mataram, Indonesia, realizaron diferentes pruebas para medir los efectos de los laboratorios virtuales en el mejoramiento de la creatividad de los futuros profesores de física, encontrando un aumento en la creatividad verbal y figurativa de los profesores que usan este tipo de herramientas [21]. Así mismo, Mirçik, Özden y Saka, Ahmet, realizaron un trabajo de capacitación focalizada que tenía por intencionalidad informar a educadores de física, respecto a la existencia de los laboratorios virtuales utilizados en la enseñanza de la física en la actualidad y el alcance y las características de estos [22]. Por otro lado, Álvarez, A. y Santoyo J. realizaron un trabajo referente a Internet de las cosas y herramientas de software libre aplicadas a la educación; en este trabajo se diseñó un prototipo de laboratorio para la implementación de actividades didácticas que apoyan el aprendizaje en diferentes ámbitos del conocimiento, haciendo uso del software Node-RED acompañado de la placa Raspberry Pi [23]. En el trabajo titulado Validación de requisitos funcionales de un Laboratorio Virtual Remoto como apoyo al blended learning, se analizó los requisitos entre estudiantes y profesores de enseñanzas científico-técnicas, sobre el diseño y funcionalidad de una plataforma web que implemente un Laboratorio Virtual Remoto (VRL) [24].

\section{Metodología de DeSARRollo}

Las metodologías de desarrollo de sistemas embebidos, toman cada día más fuerza; puesto que no solo se trata de desarrollar software y hardware de calidad, sino que estos a su vez se deben integrar a través de protocolos y sistemas eficientes de comunicación [25], [26]. Para este caso se asume como sistema embebido a una aplicación de propósito específico, alojada en un procesador o en un dispositivo programable, a través del cual se maneja información generada por sensores y por medio de señales de salida, retroalimentar la posición de actuadores para realizar acciones de control o simplemente hacer el despliegue de información para cuestiones de monitoreo [27].

Para la materialización de este proyecto, se analizaron diferentes metodologías de desarrollo ágil, (Agile Software Development Cicle o SLDC) [28], [29]; que, si bien estas surgen y son utilizadas en el desarrollo de software, gran parte de las técnicas ágiles pueden ser utilizadas en la construcción de sistemas embebidos. Sin embargo cabe anotar que una vez revisado el estado del arte, no se halló una metodología ampliamente aceptada para el desarrollo integral de este tipo de procedimientos [26], [30]; por consiguiente se trabajó con una Metodología Scrum[31], para guiar el proceso de construcción del software, esta a su vez se integró con una metodología de modelo en $\mathrm{V}$ (procedimiento uniforme para el desarrollo de productos TIC y el perfeccionamiento de software embebido [27]). En cuanto al modelo en $\mathrm{V}$, si bien es una herramienta bastante útil para guiar el desarrollo de proyectos, es posible adicionar algunos elementos que permiten precisar con mayor nivel de detalle las herramientas a utilizar en cada fase del producto, a fin de obtener resultados más confiables y de mayor calidad dentro de los límites de tiempo aceptables; en este sentido, la etapa de validación del modelo, se trabajó bajo los parámetros establecidos por el estándar IEEE 1012-2016, el cual instaura el procedimiento para realizar la verificación y validación de sistemas, software y hardware [32], como se observa en la Fig. 1.

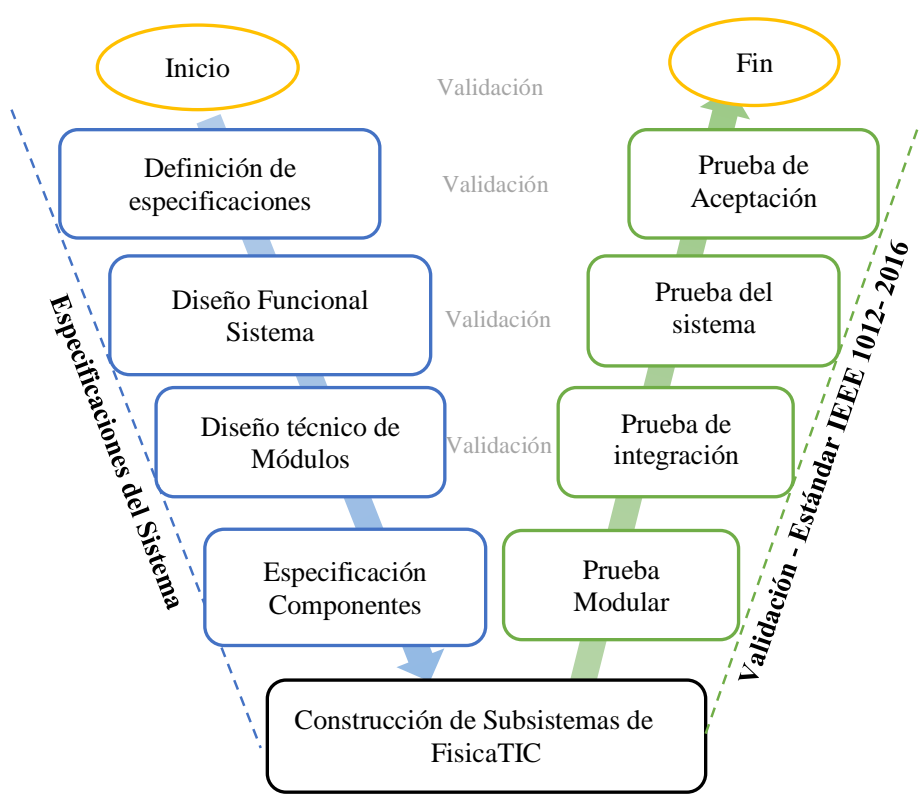

Fig. 1. Metodología utilizada para el Desarrollo del Proyecto

\section{DISEÑO}

\section{A. Definición de especificaciones}

En esta fase se definieron y documentaron los requisitos del sistema, identificando las posibles aplicaciones y laboratorios a desarrollar. Así mismo se realizó la planificación del proyecto, evaluando tecnologías existentes, protocolos de comunicación, coste, tiempos, modularidad y confiabilidad del sistema. Por otra parte, se organizó el equipo de trabajo en tres grupos para el desarrollo de los subsistemas.

\section{B. Diseño funcional del sistema}

Se definió la arquitectura general del sistema, funcionalidades, especificaciones de firmware y software; así mismo se realizó un análisis de compatibilidad e interoperabilidad del sistema, 


\section{Diseño técnico de los Módulos}

La arquitectura de FisicaTIC es modular y consta básicamente de tres subsistemas, los cuales se subdividen en diferentes unidades, como se describen a continuación:

1) Subsistema hardware: se compone de cuatro subsistemas que son: a) Unidad central de procesamiento CPU -Central processing unit-, b) unidad de comunicaciones inalámbricas, c) unidad de medición de variables del sistema y d) unidad de suministro de energía.

2) Subsistema de Aplicación para usuario final: (Plataforma web y aplicativo móvil para conexión con hardware FisicaTIC)

\section{3) Subsistema físico que está siendo monitoreando y} evaluando:

\section{Especificación de Componentes}

Referente al hardware, se tuvo en cuenta criterios de diseño tales como: desempeño, facilidad de uso, disponibilidad de componentes en el mercado (Comercial-Off-The- Shelf: COTS) y costos de implementación. Por su parte la comunicación hardware- software se implementó mediante dispositivos Bluetooth y USB-OTG, teniendo en cuenta las posibilidades de hardware y software disponibles.

Referente al software se definieron 5 pantallas principales, a través de las cuales se realizarán los procesos de autenticación de usuarios, conexión al hardware, desarrollo de laboratorios, e informes.

En la Fig. 2 se muestra un diagrama de componentes del sistema

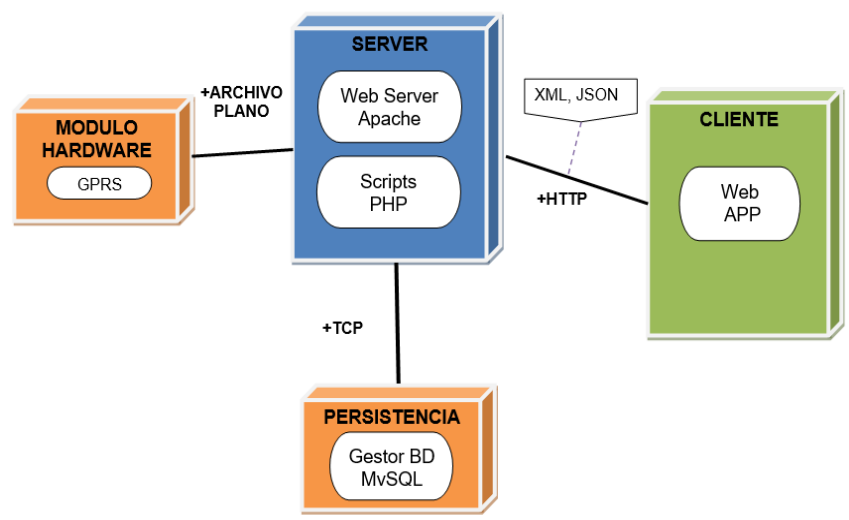

Fig. 2. Diagrama de Componentes de FisicaTIC.

\section{CONSTRUCCIÓN DE SUBSISTEMAS}

\section{A. Desarrollo del Subsistema Hardware.}

La capa de hardware se compone de cuatro subsistemas, 1) Unidad central de procesamiento CPU -Central processing unit-, 2) unidad de comunicaciones inalámbricas, 3) unidad de medición de variables del sistema y 4) unidad de suministro de energía. Para desarrollar el hardware se tuvo en cuenta como criterio fundamental en la selección de los componentes el bajo costo, buscando maximizar los resultados con una mínima inversión. En la Fig. 3 se aprecia un diagrama general de los subsistemas que componen esta capa.

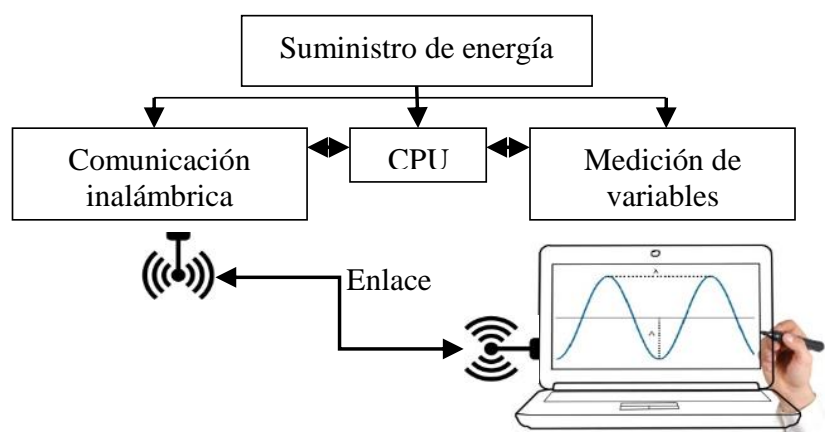

Fig. 3. Diagrama de subsistemas que componen la capa de hardware.

Asimismo, las rutinas ejecutadas por la CPU son relativamente sencillas y consisten en hacer mediciones de las aceleraciones en los ejes, $\mathrm{X}, \mathrm{Y}$ y Z, que se dan en un espacio tridimensional representado mediante coordenadas cartesianas; estas aceleraciones son experimentadas por el sistema físico compuesto por una masa suspendida en una cuerda; de este modo, en la Fig. 4 se aprecia el diagrama de flujo del algoritmo que debe ser ejecutado por la CPU, aunque de forma muy general.

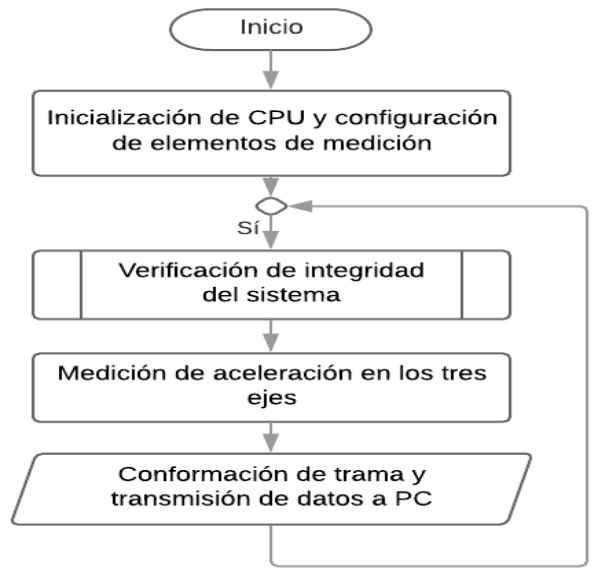

Fig. 4. Algoritmo ejecutado por la CPU del sistema propuesto

Unidad central de procesamiento. Se trabajó con el PIC12F675, en encapsulado SOIC de 8 pines, el cual, a pesar de su reducido costo, tiene características que le permiten ser adecuado para múltiples aplicaciones [33]. El algoritmo programado comprende la lectura del sensor tipo microelectromecánico Micro-Electro-Mechanical Systems (MEMS), la verificación de la integridad del sistema, suministro de energía a los componentes involucrados y el adecuado intercambio de datos con la aplicación que es ejecutada por un computador o una Tablet ya que la comunicación sistema-PC se realiza mediante un enlace inalámbrico que permite el intercambio de datos en tiempo real.

Dispositivo para medición de variables del sistema. Se trabajó con el acelerómetro MMA8452Q de la empresa 
"Freescale semiconductor" teniendo en cuenta los criterios de costo, masa y rendimiento; este es un dispositivo de bajo consumo de energía, que está diseñado para medir la aceleración en los tres ejes [34]. El aspecto de este dispositivo y sus ejes de operación se pueden apreciar en la Fig. 5.
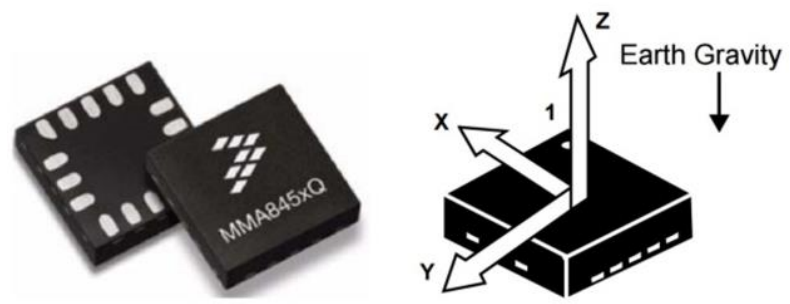

Fig. 5. Aspecto de dispositivo MMA8452Q y ejes de operación.

El MMA8452Q trabaja con velocidades de medición de entre 1.56 y 800 muestras por segundo, que pueden ser configuradas por el usuario en dependencia de su orientación espacial y del efecto gravitatorio de la tierra, sin embargo, no solo mide la aceleración gravitatoria, sino que puede medir cualquier aceleración a la que sea expuesto el cuerpo del dispositivo. Adicionalmente, existe la posibilidad de determinar la aceleración a la que es expuesto su cuerpo en cualquier eje, de forma compuesta, es decir, en cualquiera de los tres ejes en un mismo instante.

Comunicación inalámbrica. Para el sistema propuesto, se hace indispensable un canal de intercambio de datos inalámbrico, debido a que tener canales de datos que empleen cables causaría que las aceleraciones experimentadas por el dispositivo se vean alteradas, inutilizando estas mediciones; de este modo, para la comunicación inalámbrica se exploraron dos alternativas, la primera corresponde al uso del módulo Bluetooth HC-06 de la empresa "Guangzhou HC Information Technology". En la Fig. 6 se muestran las dos soluciones implementadas, en la Fig. 6 se muestran las dos soluciones implementadas, a) se aprecia el aspecto del módulo HC-06 y b) el aspecto del módulo USB HC-12.

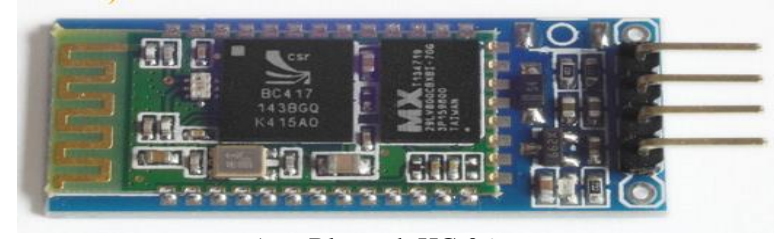

a) Bluetooh $\mathrm{HC}-06$

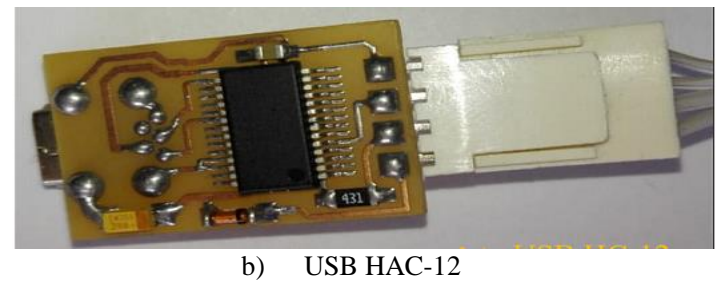

Fig. 6. a). Aspecto de módulo Bluetooth HC-06, b) aspecto de modulo USB HC-12, utilizados.

Aunque el fabricante indica que el módulo operará sin problema, siempre que la distancia del enlace no sea superior a 10 metros, las pruebas empíricas mostraron que en buenas condiciones y con línea de vista se pueden lograr distancias de hasta 100 metros; sin embargo, se debe señalar que las distancias a las que va a ser aplicado el sistema, difícilmente superarán una separación de 5 metros entre la fuente y el destino de la señal de radiofrecuencia.

Las ventajas que ofrece el uso del módulo HC-06 reside en el hecho de que en la actualidad es frecuente que una Tablet, celular o computadora tenga incorporado un módulo Bluetooth interno, bajando costos y permitiendo conectar el sistema a cualquier tipo de dispositivo de computo contemporáneo; sin embargo, las pruebas empíricas demostraron que existen algunos errores de compatibilidad con algunos equipos, a causa de las versiones del software y del hardware del que disponen; por estas razones, como medio alternativo de enlace, se dispuso del empleo de un módulo adicional de comunicaciones que permite establecer un enlace inalámbrico en el que los datos ingresan a la computadora por el puerto USB -Universal Serial Bus- para este cometido, se emplea el módulo HC-12, también de la empresa "Guangzhou HC Information Technology"; su ventaja principal, radica en que el enlace inalámbrico establecido no depende de las versiones de hardware y de software del ordenador empleado, la principal desventaja reside en el hecho de que se requiere un módulo de comunicación adicional en el lugar de la computadora, como se indica en la Fig. 7, haciendo que los costos se eleven levemente, transgrediendo el criterio del bajo costo, pero mejorando ostensiblemente la funcionalidad.

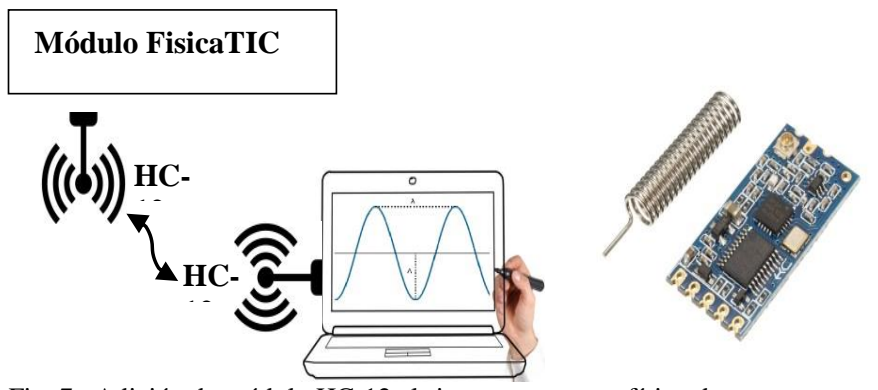

Fig. 7. Adición de módulo HC-12 al sistema y aspecto físico de este dispositivo.

De este modo, se mejora ostensiblemente la capacidad del enlace constituido por el módulo Bluetooth HC-06; el uso de uno u otro dependerá de las circunstancias particulares de cada aplicación y será el usuario final el que determine qué tipo de enlace le es más conveniente y acorde a sus necesidades.

Suministro de energía. De acuerdo con los datos suministrados por los fabricantes de los dispositivos empleados en el sistema, es posible construir la TABLA I, que corresponde a los requerimientos energéticos de cada uno de los subsistemas que componen la capa de hardware.

Se seleccionó una batería recargable de iones de litio referencia 18650 de $4000 \mathrm{~mA} / \mathrm{h}$, ya que este acumulador suministra un voltaje de 3.7 voltios, es de fácil consecución en el mercado y ofrece una excelente relación masa/energía acumulada; adicionalmente, con esta fuente de energía no se requieren reguladores de voltaje extras, aumentando la eficiencia del sistema; las pruebas empíricas demostraron que se puede 
mantener funcionando la capa de hardware de forma continua alrededor de 100 horas con el módulo HC-06 y cerca de 30 horas con el módulo HC-12, antes de una nueva recarga.

TABLA I

REQUERIMIENTOS ENERGÉTICOS DE LOS ELEMENTOS CONSTITUTIVOS DEL SISTEMA

\begin{tabular}{lll}
\hline \hline \multicolumn{1}{c}{ Item } & $\begin{array}{c}\text { Rango de voltaje de } \\
\text { operación }\end{array}$ & $\begin{array}{c}\text { Máxima corriente } \\
\text { requerida }\end{array}$ \\
\hline Pic12f675B & $2.0 \mathrm{~V}-5.5 \mathrm{~V}$ & $10 \mathrm{~mA}$ \\
Mma $8452 q$ & $1.95 \mathrm{~V}-3.7 \mathrm{~V}$ & $0.5 \mathrm{~mA}$ \\
Hc-06 & $3.1 \mathrm{~V}-4.2 \mathrm{~V}$ & $5 \mathrm{~mA}$ \\
Hc-12 & $3.2 \mathrm{~V}-5.5 \mathrm{~V}$ & $16 \mathrm{~mA}$ \\
\hline \hline
\end{tabular}

$\mathrm{mA}=$ miliamperios, $\mathrm{V}=$ voltios

Circuito impreso. El sistema debe ajustarse mecánicamente a un gabinete que lo contenga, para alcanzar este objetivo, es necesario construir un PCB -Printed Circuit Board- que de soporte mecánico y conectividad eléctrica a cada uno de los elementos que componen el sistema; los criterios para la construcción de este PCB son: a) bajo costo, b) masa mínima y c) soporte adecuado para cada dispositivo; de esta forma, se realizó el diseño de este PCB mediante el software Eagle 5.7.0 versión libre; el resultado del procedimiento descrito aparece en la Fig. 8 en donde se observa el diseño realizado mediante el software y el aspecto de la tarjeta en baquelita, previo a la etapa en la que se soldaron los componentes.

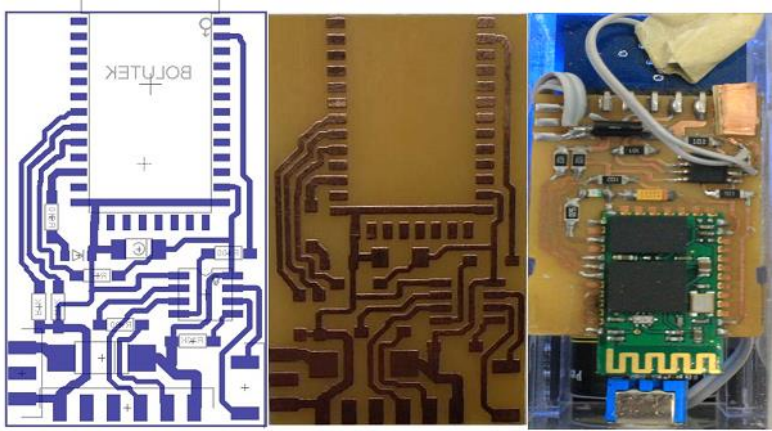

Fig. 8. Diseño de PCB y aspecto final de baquelita y circuito

\section{B. Desarrollo del Subsistema Software.}

Para el desarrollo de la aplicación Android se utilizó Scrum, el cual es un proceso de la Metodología Ágil que se usa para minimizar los riesgos durante la realización de un proyecto; y como herramienta de programación se trabajó con Basic4Android. Por su parte la codificación se realizó de acuerdo a los cinco módulos definidos en la etapa de diseño, realizando pruebas continuas de funcionamiento y ajustes en cada módulo desarrollado, para posteriormente integrarlos con el hardware y obtener un software con todas las funcionalidades y prestaciones.

La aplicación desarrollada permite la comunicación con el módulo FisicaTIC a través de Bluetooth o USB (protocolo OTG). Se diseñaron 5 pantallas principales: Intro, Menú de Temas, Menú de Subtemas, Menú Opciones Laboratorio. En este último se puede encontrar la guía del laboratorio, el panel de control principal y el formulario del informe.

En el panel de control se encuentran las opciones para realizar la conexión con el módulo FisicaTIC, registrar datos en archivo plano(CVS), capturar la pantalla (evidencias para el informe final) y habilitar la cámara del dispositivo para tomar evidencias del desarrollo del laboratorio.

Como parte del procesamiento de datos, una vez recibidos por el puerto Bluetooth o USB, estos se ajustan de acuerdo al área de visualización y se van graficando en tiempo real.

Los datos almacenados en el archivo plano, permiten realizar los cálculos de ecuaciones para comprobar las temáticas del laboratorio a desarrollar.

\section{Descripción de pantallas}

Pantalla de bienvenida. se muestra al abrir la aplicación, como se observa en la Fig. 9.

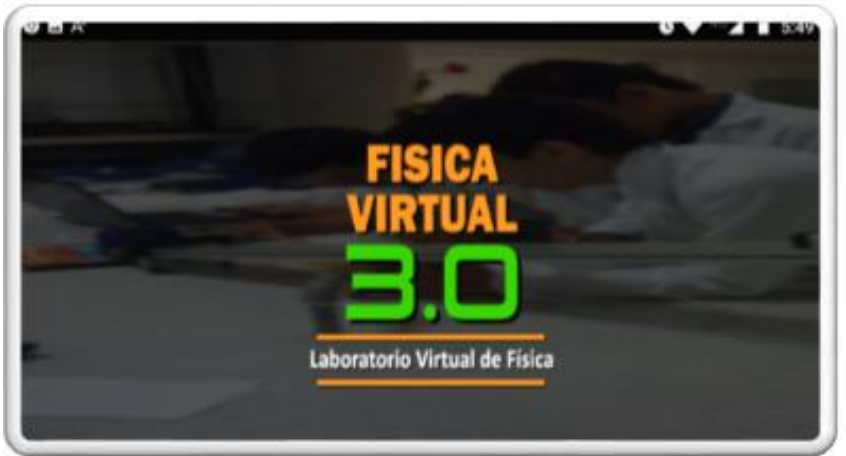

Fig. 9. Pantalla de Bienvenida de FisicaTIC

Menú Principal. En esta pantalla se pueden incluir los diferentes temas asociados a los laboratorios de física. Para efectos de pruebas se estableció el tema de Movimiento Armónico. Este menú se muestra en la Fig. 10.

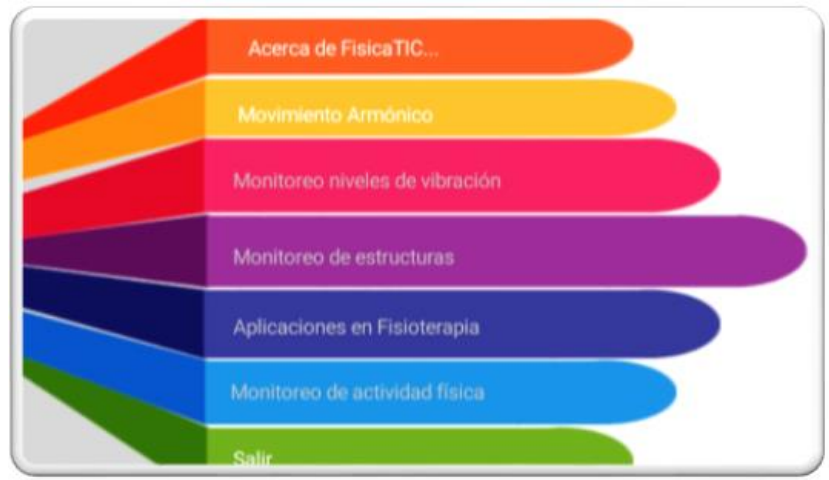

Fig. 10. Vista del Menú Principal de FisicaTIC

Menú de Subtemas. Muestra los contenidos temáticos, de acuerdo al tema seleccionado en la pantalla anterior (ver Fig. 11). Para este caso el Péndulo Simple. 


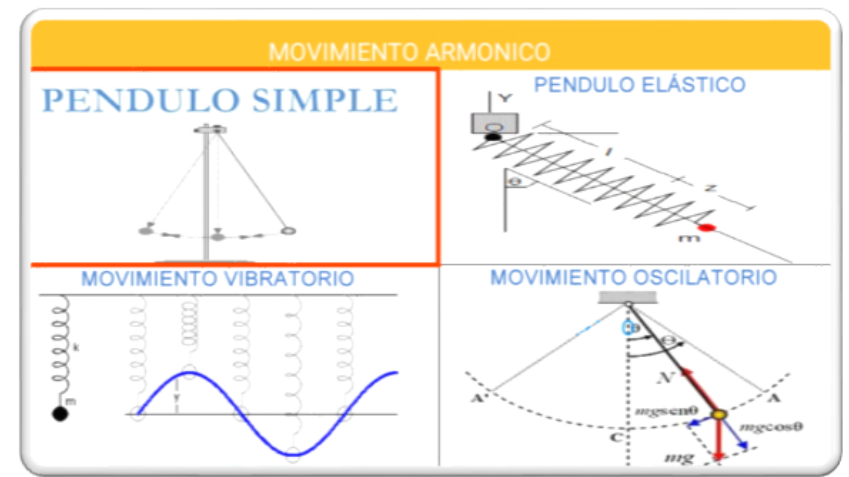

Fig. 11. Vista del Menú Subsistemas de FisicaTIC

Menú Laboratorio. El usuario podrá encontrar la guía de laboratorio desarrollada, acceder al panel de control y diligenciar el formulario del informe (ver Fig. 12).

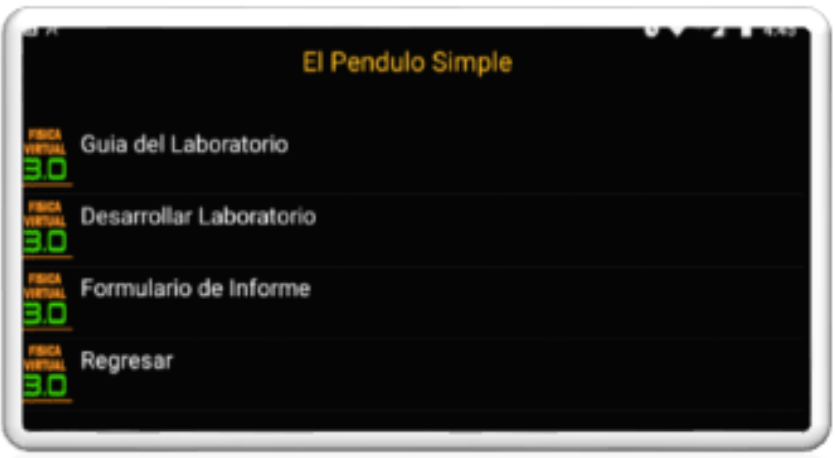

Fig. 12. Vista Menú Laboratorios

Panel de Control. Esta pantalla permite gestionar las diferentes funciones como, realizar la conexión con el módulo FisicaTIC, registrar datos en archivo plano(CVS), capturar la pantalla (evidencias para el informe final) y habilitar la cámara del dispositivo para tomar evidencias del desarrollo del laboratorio (ver Fig. 13.)

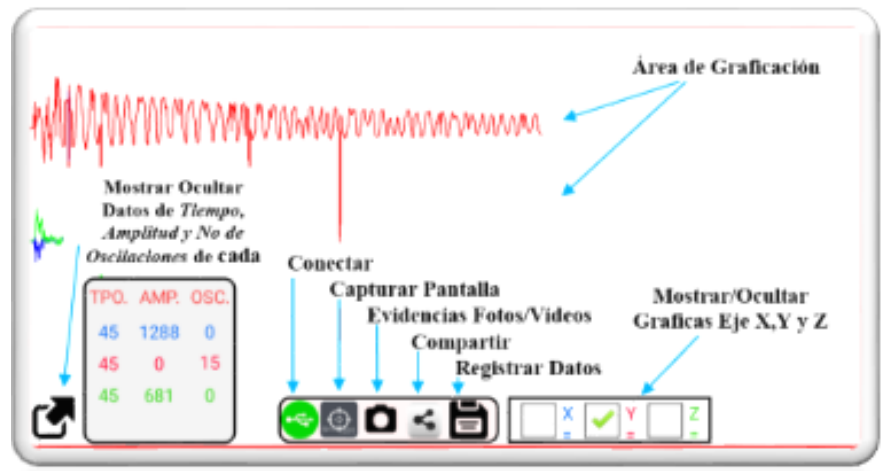

Fig. 13. Vista Panel de Control de FisicaTIC

Las pruebas generales de la App se realizaron sobre el dispositivo móvil, estableciendo la comunicación con el módulo FisicaTIC, con el fin de realizar el tratamiento de los datos, medir las funcionalidades, robustez de la App y con cada falla identificada, reajustar el código hasta ponerlo a punto. Para este proceso se desarrollaron varios módulos internos que se describen a continuación:

\section{Bloque Comunicación Bluetooth}

A continuación, se describe el proceso de programación realizada para lograr la comunicación mediante el estándar Bluetooth IEEE 802.15.1. Para esta función se utilizó la librería Serial de Basic4Android, la cual permite buscar dispositivos Bluetooth, determinar los dispositivos previamente conectados, abrir canales de comunicación y enviar y recibir datos con el dispositivo seleccionado.

Una vez se establece la comunicación, durante el proceso de detección de nuevo dato en el puerto serial Bluetooth, un proceso (Thread) es asignado para escuchar el puerto serial Bluetooth. Cuando se detecta un nuevo paquete de información se utiliza el ultimo byte para identificar si la trama de datos es correcta. Si es correcta, esta trama se decodifica y procesa de acuerdo a las especificaciones del acelerómetro. La trama original está representada por una cadena de 7 bytes en formato ASCII, que debe procesarse y convertirse en forma decimal.

\section{Bloque Comunicación USB}

En este proceso se utilizó la librería UsbSerial Versión 2.30, que permite determinar el dispositivo conectado al puerto OTG, obtener sus especificaciones, abrir el canal de comunicación, enviar y recibir tramas de datos. Para establecer comunicación con el módulo FisicaTIC es necesario establecer la velocidad a 9600 baudios.

\section{Módulo Gráfico}

Encargado del procesamiento y visualización de los datos recibidos. Teniendo en cuenta los datos de las coordenadas $\mathrm{X}$, Y y Z; se inicia el proceso de graficación, en el cual se establecen las coordenadas iniciales y finales para trazar una línea de conexión y de esta forma ir generando la gráfica de la señal recibida, con el nuevo dato recibido. Lo anteriormente mencionado se aprecia en la Fig. 14.

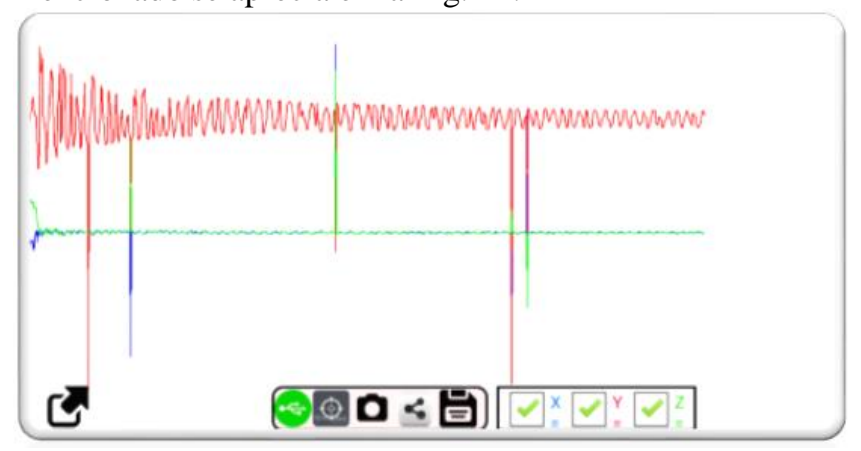

Fig. 14. Módulo Gráfico

\section{Módulo determinador de oscilaciones}

Para determinar las oscilaciones se analizó una trama de datos posteriores al proceso de adecuación de señal, en la que el dato se encuentra definidos de la forma $\left(x 1, y 1_{x}\right) y\left(x 2, y 2_{-} x\right)$. Teniendo en cuenta este punto, se analizó el valor $y 2 \_x$, determinando el valor mínimo y máximos, en este punto la onda de la gráfica comienza a crecer y cuando $y 2 \_x$, llegaba a su valor máximo y empezaba a decrecer, se establecía un cambio descendente de la oscilación. De esta forma se identificaron las oscilaciones para poder realizar el conteo de las mismas. 


\section{Módulo de almacenamiento}

Este módulo se encarga de registrar los datos generados por el acelerómetro, para que posteriormente puedan ser analizados, procesados o generar informes a partir de los mismos. Como medio de almacenamiento se utilizó un sistema archivo plano, de tal forma que se pudiera compartir o utilizar fácilmente o exportarlos por ejemplo a Excel.

Para esto se creó el método escribir Archivo, haciendo uso de la función

File.WriteString(File.DirRootExternal, "acelerometro.txt",txt\& $C R L F \&$ dato \&CRLF)

De la misma manera se creó el método para realizar la posterior lectura de los datos almacenados, haciendo uso de la siguiente instrucción:

File.ReadString(File.DirRootExternal,"acelerometro.txt")

\section{VALIDACIÓN Y PRUEBAS DEL SISTEMA}

Las pruebas del sistema se realizaron según la Norma IEEE 1012- 2016, la cual establece que los procesos de verificación y validación $(\mathrm{V} \& \mathrm{~V})$ se utilizan para determinar si los productos de desarrollo de una actividad determinada cumplen con los requisitos y si el producto satisface su uso previsto y las necesidades del usuario [32]. Esta norma se aplica a los sistemas, software y hardware que se desarrollan, utilizando componentes disponibles en el mercado Comercial Off-TheShelf (COTS). En la TABLA II, se describen las principales pruebas según el estándar IEEE 1012- 2016 y la metodología planteada para el desarrollo del proyecto.

TABLA II

Pruebas Realizadas SegúN El ESTÁNDAR IEEE 1012- 2016

\begin{tabular}{lll}
\hline \hline No & \multicolumn{1}{c}{ Ítem } & \multicolumn{1}{c}{ Descripción } \\
\hline 1 & Prueba modular & $\begin{array}{l}\text { Prueba de cada módulo aislado del } \\
\text { resto del sistema }\end{array}$ \\
2 & Prueba de integración & $\begin{array}{l}\text { A medida que los diferentes } \\
\text { módulos del sistema se integran }\end{array}$ \\
3 & Prueba del sistema & $\begin{array}{l}\text { Comprueba que el sistema satisface } \\
\text { los requisitos del usuario }\end{array}$ \\
4 & Prueba de aceptación & $\begin{array}{l}\text { Una vez que el sistema se ha } \\
\text { implantado en su entorno real }\end{array}$ \\
5 & Prueba de regresión & $\begin{array}{l}\text { Comprobar que nueva versión sea } \\
\text { de mejor calidad que la anterior }\end{array}$ \\
\hline \hline & Tabla realizada según estándar IEEE 1012- 2016 [32]
\end{tabular}

a) Prueba modular: "consiste en la prueba de cada módulo aislado del resto del sistema". Cada grupo de trabajo por separado realizo las pruebas de los distintos subsistemas, validando su correcto funcionamiento y revisando la interoperabilidad.
- Subsistema hardware: se realizaron pruebas del funcionamiento de la CPU, revisando la funcionalidad del microcontrolador, del acelerómetro y de la unidad de suministro de energía.

- Subsistema de comunicación inalámbrica: se realizaron pruebas de protocolos de comunicación en sistemas embebidos, analizando tecnologías Bluetooth inicialmente, sin embargo, se encontraron varias dificultades en los tiempos de transmisión con algunos dispositivos electrónicos, lo cual afectaba notablemente los tiempos de graficación por lo que fue necesario implantar paralelamente un módulo USB-OTG para eliminar este problema. Finalmente, el sistema quedo implementado con las dos formas de comunicación.

- Aplicación para usuario final. Se realizaron pruebas de funcionalidad de los cinco módulos, (Intro, Menú de Temas, Menú de Subtemas, Menú Opciones Laboratorio). En cuanto a App se realizaron pruebas sobre dispositivos móviles, estableciendo la comunicación con el módulo FisicaTIC, con el fin de realizar el tratamiento de los datos, medir las funcionalidades, robustez de la App y con cada falla identificada, reajustar el código hasta ponerlo a punto.

b) Prueba de integración: "se realiza a medida que los diferentes módulos del sistema se integran". El objetivo fundamental de esta prueba es comprobar que las interfaces entre los distintos módulos son correctas. En esta etapa se realizó la integración de los subsistemas, hardware y software, a través del sistema inalámbrico de comunicación el cual recibe los datos del sistema físico, los adapta y transmite a la aplicación de Usuario Final. En cuanto a la integración física de las tarjetas se diseñó una caja modular transparente afín de disminuir interferencias y asegurar las tarjetas, tal como se observa en la Fig. 15.

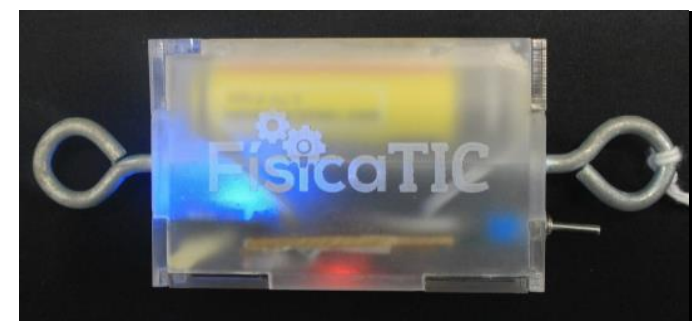

Fig. 15. Vista frontal, integración hardware y tarjeta de comunicación.

c) Prueba del sistema: "se realiza cuando se han integrado todos los módulos". su objetivo es comprobar que el sistema satisface los requisitos del usuario, tanto los funcionales como los no funcionales.

En este sentido se elaboraron dos prototipos similares, uno con los dos sistemas de comunicación implementados (Bluetooth y USB-OTG) y el otro únicamente con Bluetooth, con el fin de comparar y realizar pruebas de conexión y funcionamiento en diferentes dispositivos electrónicos.

Dentro de las pruebas realizadas al sistema se revisó latencias, retardos de transmisión, almacenamiento, registro de datos y capacidad de carga de la unidad de suministro de energía. Por otra parte, en el software se 
revisó, el funcionamiento de los módulos, tiempos de conexión con el hardware y realizaron ajustes de los módulos de graficación y presentación de informes.

Con la ejecución de todas estas pruebas, se realizaron los ajustes el sistema en conjunto y se pudo establecer que el sistema Bluetooth presenta inconvenientes en el proceso de conexión y retardos significativos en el proceso de transmisión con algunos dispositivos electrónicos; mientras que con la tarjeta USB-OTG, se eliminan estos inconvenientes. En la Fig. 16 se muestra el proceso de validación y pruebas del sistema, en este caso se instaló un soporte metálico, al cual se sujetó el hardware de FisicaTIC de forma perpendicular para realizar los movimientos en los tres ejes X, Y y Z. El software se puede descargar desde https://fisicatic.com/

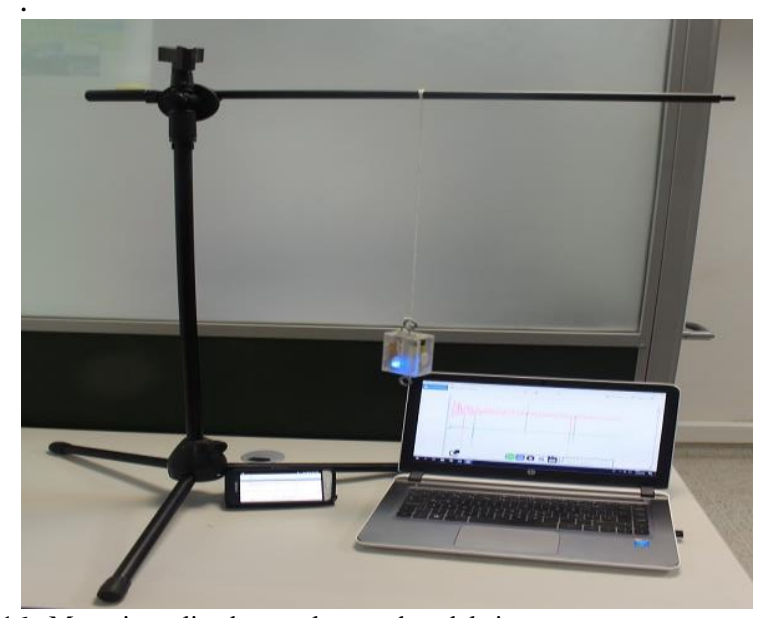

Fig. 16. Montaje realizado para las pruebas del sistema

d) Prueba de aceptación: "se realiza una vez que el sistema se ha implantado en su entorno real de funcionamiento"; su objetivo es demostrar al usuario que el sistema satisface sus necesidades.

Las pruebas de aceptación finales del prototipo se llevaron a cabo, realizando el laboratorio del péndulo simple, el cual consiste básicamente en demostrar la relación entre el periodo de oscilación $T$ y la Longitud del Péndulo $L$, y calcular experimentalmente la aceleración gravitacional local $g$. Para este caso se realizó una prueba real del laboratorio del péndulo simple, tal como lo realizan los alumnos de ciencias básicas e ingeniería de Unitrópico, utilizando el Xplorer GLX, este es un sistema informático de mano, totalmente autónomo que cuenta con sensores de movimiento para pruebas de laboratorios de ciencias básicas entre ellos el péndulo simple. Paralelamente se realizó el mismo laboratorio con el módulo FisicaTIC, tal como se muestra en la Fig. 17.

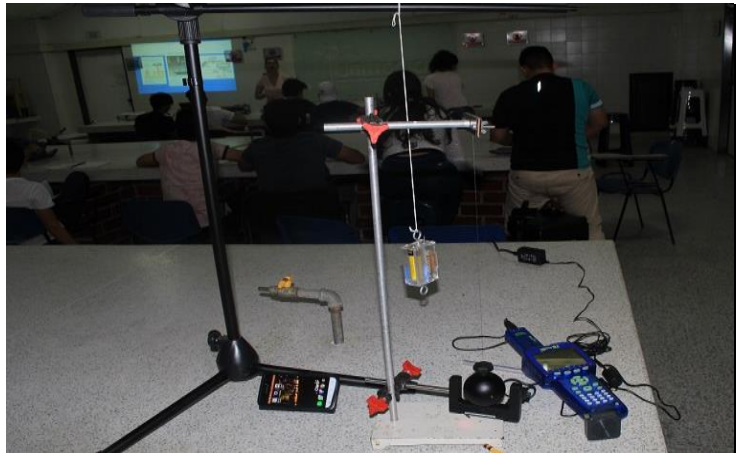

Fig. 17. Montaje Laboratorio Péndulo Simple

Para desarrollar el laboratorio se tuvieron en cuenta las siguientes condiciones para ambos dispositivos: Angulo de separación del hilo de la vertical $=10^{\circ}$ Masa suspendida $=2 \mathrm{~kg}$ Longitud del hilo $=40 \mathrm{~cm}$

La práctica realizada con el Xplorer GLX obtuvo los siguientes datos:

TABLA III

DATOS OBTENIDOS CON EL XPLORER GLX

\begin{tabular}{lccc}
\hline \hline No & Periodo (s) & Tiempo(s) & Velocidad $(\mathrm{m} / \mathrm{s})$ \\
\hline 1 & 2,7320 & 36,53 & 0.02 \\
2 & 2,7375 & 39,26 & 0.02 \\
3 & 2,7353 & 42,00 & 0.02 \\
4 & 2,7307 & 44,73 & 0.02 \\
5 & 2,7368 & 47,46 & 0.02 \\
\hline \hline
\end{tabular}

TABLA IV

DATOS OBTENIDOS CON FISICATIC

\begin{tabular}{lll}
\hline \hline No & \multicolumn{1}{c}{ Periodo (s) } & \multicolumn{1}{c}{ Tiempo(s) } \\
\hline 1 & 2,7294 & 4,29 \\
2 & 2,7275 & 7,02 \\
3 & 2,7297 & 9,76 \\
4 & 2,7301 & 12,49 \\
5 & 2,7292 & 15,22 \\
\hline \hline
\end{tabular}

De acuerdo a los datos obtenidos en las tablas III y IV se puede observar que el periodo de ambos dispositivos es similar, $\mathrm{T}=\mathbf{2 . 7 3}$ sin embargo, en los datos de FisicaTIC se observa que las oscilaciones se estabilizan en menor tiempo 4,29 segundos, mientras que en el Xplorer GLX, se estabilizan en 36 segundos aproximadamente. Esto permite inferir que FisicaTIC al no depender de sensor externos permite medir oscilaciones con ángulos más pequeños, mientras que el Xplorer GLX depende del tamaño de la masa y de la ubicación del sensor. Por otra parte, FisicaTIC permite medir las oscilaciones en los otros dos ejes de forma paralela, esto permite hacer estudios más precisos dado que se puede correlacionar la afectación de los movimientos en los otros ejes, lo cual no es posible con el Xplorer GLX.

e) Prueba de regresión: tiene como objetivo comprobar que toda nueva versión de un producto software es de no menos 
calidad que la versión anterior, es decir, que al introducir cambios no se ha reducido la valoración de ninguna de las características de calidad que tenía el producto. Esta prueba no aplica para este caso dado que es una primera versión.

\section{Resultados FinALES}

\section{Pruebas}

Como se describió en la sección de pruebas, los tres subsistemas (Unidad Central de Procesamiento, Módulo de comunicación y Plataforma web /APP), funcionaron eficientemente a nivel individual y en la etapa de integración. Respecto al hardware desarrollado se ajusta a las necesidades requeridas, dado que permite capturar la información en los tres ejes X, Y y Z y transmite a la aplicación sin ningún contratiempo.

Por otro lado, los materiales utilizados en este desarrollo son de fácil consecución en el mercado y económicos.

Respecto a la aplicación web y la APP, funcionaron correctamente, logrando comunicarse eficiente con el hardware desarrollado, permitiendo observar en tiempo real los datos obtenidos por el acelerómetro, a través de una interfaz gráfica de usuario, amigable y entendible; gracias al diseño que puede adaptarse fácilmente a diferentes plataformas tecnológicas: Ordenador, Tablet y Smartphone.

La validación y comparación de FisicaTIC, con otras herramientas, permitió ajustar el sistema, de forma tal que se minimizo al máximo la posibilidad de fallos, gracias a las diferentes pruebas; esto es muy importante porque garantiza una confiabilidad en el dispositivo, pensando en una patente y comercialización exitosa.

Por otra parte, se realizó un análisis integral de los resultados obtenidos del funcionamiento del hardware, del software y de los dos sistemas de comunicación implementados; comprobando que se cumplió a cabalidad con el objetivo planteado para este proyecto.

\section{Comparativas}

A fin de comparar el sistema descrito en este artículo, con otros revisados en la sección de Antecedentes, se definió un enfoque técnico, utilizando como factor tecnológico comparativo: sensores, CPU, protocolos de comunicación, almacenamiento de información y plataforma web/APP. En la Tabla V se muestran los resultados de esta comparación.

Como se puede observar en la tabla V, el módulo FisicaTIC posee ciertas ventajas frente a los dispositivos relacionados. El caso de Xplorer GLX es un dispositivo costoso que requiere de muchas conexiones y para el laboratorio del péndulo permite analizar solo dos ejes debido al sensor que utiliza. En cuanto PhysicsSensor hay una gran similitud con FisicaTIC, en cuanto a que hace uso de los equipos electrónicos (PC, Celular o Tablet) de los estudiantes para realizar los laboratorios, disminuyendo los costos, sin embargo, utiliza la tarjeta ARDUINO que, aunque es muy buena, siempre se dependerá estrictamente de las características electrónicas de esta placa base.
TABLA V

COMPARACIÓN TECNOLÓGICA DE FISICATIC, XPLORER GLX Y PHYSICSSENSOR

\begin{tabular}{|c|c|c|c|}
\hline $\begin{array}{c}\text { Factor } \\
\text { comparativo }\end{array}$ & $\begin{array}{c}\text { Xplorer GLX } \\
{[10]}\end{array}$ & $\begin{array}{c}\text { PhysicsSensor } \\
{[9]}\end{array}$ & FisicaTIC \\
\hline $\begin{array}{l}\text { Micro- } \\
\text { controlador }\end{array}$ & $\begin{array}{l}\text { FPU de } 50 \\
\text { MHz: }\end{array}$ & $\begin{array}{l}\text { ARDUINO, } \\
\text { Microcontrolad } \\
\text { or: } \\
\text { ATmega328. }\end{array}$ & $\begin{array}{l}\text { PIC12F675, en } \\
\text { encapsulado } \\
\text { SOIC de } 8 \text { pines }\end{array}$ \\
\hline Sensores & $\begin{array}{l}\text { Se venden } 8 \\
\text { en Total, } \\
\text { sonido, } \\
\text { Voltaje } \\
\text { Temperatura, } \\
\text { conexión } \\
\text { cableada }\end{array}$ & $\begin{array}{l}\text { Acelerómetro } \\
\text { inclinómetro, } \\
\text { termómetro } \\
\text { barómetro, } \\
\text { higrómetro. }\end{array}$ & $\begin{array}{l}\text { acelerómetro } \\
\text { MMA8452Q, } \\
\text { incluido dentro } \\
\text { del hardware, } \\
\text { conexión } \\
\text { inalámbrica }\end{array}$ \\
\hline $\begin{array}{l}\text { Protocolo de } \\
\text { comunicación }\end{array}$ & $\begin{array}{l}\text { USB y } \\
\text { conexión } \\
\text { alámbrica } \\
\text { serial }\end{array}$ & $\begin{array}{l}\text { Tarjeta de } \\
\text { adquisición de } \\
\text { datos de } \\
\text { ARDUINO }\end{array}$ & $\begin{array}{l}\text { Soporta Puertos } \\
\text { USB OTG, } \\
\text { Bluetooth }\end{array}$ \\
\hline $\begin{array}{l}\text { Almacenamiento } \\
\text { de información }\end{array}$ & $\begin{array}{l}12 \mathrm{MB} \text { de } \\
\text { Memoria } \\
\text { interna, }\end{array}$ & $\begin{array}{l}\text { Almacenamient } \\
\text { o externo en } \\
\text { dispositivo } \\
\text { conectado (PC, } \\
\text { Tablet o } \\
\text { Celular) }\end{array}$ & $\begin{array}{l}\text { Almacenamiento } \\
\text { externo en } \\
\text { dispositivo } \\
\text { conectado (PC, } \\
\text { Tablet o Celular) }\end{array}$ \\
\hline Plataforma Web & $\begin{array}{l}\text { Licencia de } \\
\text { Software, por } \\
\text { aparte. } \\
\text { LINUX, Mac, } \\
\text { Windows. }\end{array}$ & $\begin{array}{l}\text { LINUX, } \\
\text { Windows, Mac } \\
\text { y Android. }\end{array}$ & $\begin{array}{l}\text { Software para } \\
\text { instalar en } \\
\text { Windows y } \\
\text { Androide. }\end{array}$ \\
\hline $\begin{array}{l}\text { Fuente de } \\
\text { Alimentación }\end{array}$ & $\begin{array}{l}\text { Recargable } \\
1700 \text { mAH } \\
\text { NiMH battery }\end{array}$ & $\begin{array}{l}\text { Voltaje de } \\
\text { Entrada } \\
\text { (Recomendado) } \\
: 7-12 \mathrm{v} .\end{array}$ & $\begin{array}{l}\text { Batería } \\
\text { recargable de } \\
18650 \text { de } 4000 \\
\mathrm{~mA} / \mathrm{h}, 3.7 \mathrm{~V} .\end{array}$ \\
\hline
\end{tabular}

Una ventaja adicional de FisicaTIC, es que cuenta con diversas formas de comunicación lo que permite hacer uso de variados dispositivos electrónicos, aunado a esto la interfaz USB-OTG permite una conexión transparente. Por otra parte, FisicaTIC al ser un módulo integrado permite realizar los laboratorios fácilmente sin necesidad de conexiones físicas de cables y artefactos que hacen dispendioso el procedimiento $\mathrm{y}$ posiblemente incrementan el error por instrumentos, al tener que utilizar muchos componentes.

\section{Confiabilidad del sistema}

Un factor muy importante para este sistema es la confiabilidad. En este sentido se optimizo el software y los protocolos de comunicación a fin de obtener la información del sensor de la forma más fácil y confiable.

\section{Otras Posibles aplicaciones}

Se pueden realizar estudios de oscilaciones en 2D y 3D, cálculos de gravedad, laboratorio de osciladores acoplados; también se puede utilizar en sistemas de monitoreo remoto de niveles de vibración en máquinas industriales y obras civiles como puentes y construcciones; sistemas de detección y medida de choque e impacto en sanidad, robótica y automatización y control de proceso de fabricación; monitoreo de estructuras al interior de minas de carbón; aplicaciones en fisioterapia y actividad física; detección de movimiento en 3D,entre otros. 


\section{CONCLUSIONES}

Aunque se da por sentado que una comunicación Bluetooth es suficiente para conectar dispositivos a cortas distancias, se debe tener en cuenta que debido a diversas incompatibilidades de hardware y de software, en algunas ocasiones, es dispendioso establecer un enlace inalámbrico fiable en tiempo real, una alternativa a tener en cuenta está representada en el uso de módulos alternos como el HC12, de bajo costo, bajo consumo y altas prestaciones; adicionalmente se debe mencionar que los módulos Xbee son una alternativa adicional, sin embargo no fueron empleados en el diseño del sistema descrito, debido a que transgreden el criterio del bajo costo, aunque con creces cumplen con los requerimientos de conectividad y bajo consumo energético requeridos.

Se debe mencionar que un procesador simple como el 12F675, aunque con unos recursos modestos, puede ejecutar algoritmos de baja y mediana complejidad, en buena medida en dependencia de la pericia y el ingenio del programador, ofreciendo como contraprestación un muy reducido costo y un limitado uso de espacio en el PCB, razón por la cual, CPU basadas en estos microcontroladores deben ser tenidas en cuenta como alternativa de solución, en eventuales circunstancias.

Los dispositivos MEM son elementos versátiles que pueden ser empleados en la solución de innumerables problemas presentes en los hogares, en las industrias y en la educación, como se ha verificado en el sistema descrito en el presente texto, permitiendo que conceptos que se abordan en la escuela, sean vistos desde nuevas perspectivas, logrando que los estudiantes subsuman en su estructura cognoscitiva, nuevas formas de conceptualizar y entender la realidad.

Los resultados obtenidos en el proceso de validación, indican que los alumnos pueden desarrollar sus habilidades cognoscitivas durante la interacción con la plataforma, propiciando la comprensión y adquisición de los conceptos relacionados con el péndulo simple, de forma práctica gracias a la sencillez con que se realizan los montajes y a la habilidad de ellos en el manejo de los dispositivos electrónicos.

La APP de FisicaTIC es una valiosa herramienta que puede ser usada en los laboratorios básicos de enseñanza de la física y la ingeniería, la cual facilita la implementación y el buen uso de las Tecnologías de la Información y las Comunicaciones en el proceso educativo, disminuyendo costos y generando valor agregado, a través de los dispositivos electrónicos de los alumnos, que se convierten en poderosos instrumentos virtuales, que generan alto conocimiento tecnológico en el proceso formativo.

De acuerdo con las validaciones realizadas frente al sistema actual utilizado para el laboratorio del péndulo simple, se evidencia una reducción en el tiempo invertido para el desarrollo del laboratorio, debido a la facilidad del montaje y a la aplicación móvil que genera automáticamente los datos del informe del laboratorio (gráficas y tablas de datos), gracias a que el teléfono celular puede capturar la pantalla y exportar directamente los datos en archivo plano, lo cual minimiza el uso de papel; ventajas que no ofrecen otros sistemas que realizan el proceso de forma manual.

FisicaTIC permite realizar estudios matemáticos más precisos debido a que registra los datos en los tres ejes de forma paralela, permitiendo trabajar con ángulos muy pequeños y en el caso del laboratorio del péndulo, permite correlacionar el efecto de los otros dos ángulos con el valor del periodo.

Esta plataforma es fácilmente aplicable en la construcción de otro tipo de soluciones tecnológicas que involucran el uso de datos de aceleración, como los sistemas de monitoreo remoto de niveles de vibración, laboratorios experimentales en ciencias naturales y física, sistemas de detección y medida de choques e impactos, robótica, automatización y control de proceso de fabricación entre otros. Esto teniendo en cuenta que los datos leídos del acelerómetro son enviados a través de comunicación Bluetooth o USB-OTG que son protocolos ampliamente utilizado en muchos dispositivos tecnológicos.

\section{REFERENCIAS}

[1] C. Viegas et al., "Impact of a remote lab on teaching practices and student learning," Computers \& Education, vol. 126, pp. 201-216, Nov. 2018. DOI: 10.1016/j.compedu.2018.07.012

[2] J E Silva and M. R. R. Jeldres, "La virtualidad una oportunidad para innovar en educación: un modelo para el diseño de entornos virtuales de aprendizaje," Revista Didasc@lia: Didáctica y Educación. ISSN 2224-2643, vol. 5, no. 1, pp. 01-22, Feb. 2018.

[3] E. A. Espinosa-Ríos, K. D. González-López, and L. T. HernándezRamírez, "Las prácticas de laboratorio: una estrategia didáctica en la construcción de conocimiento científico escolar," Revista Entramado, vol. 12, no. 1, pp. 266-281, 2016. DOI:10.18041/entramado.2016v12n1.23125

[4] L. D. Feisel and A. J. Rosa, "The role of the laboratory in undergraduate engineering education," in Journal of Engineering Education, 2005, vol. 94, no. 1, pp. 121-130. DOI: 10.1002/j.21689830.2005.tb00833.x

[5] R. Heradio, L. de la Torre, D. Galan, F. J. Cabrerizo, E. HerreraViedma, and S. Dormido, "Virtual and remote labs in education: A bibliometric analysis," Computers \& Education, vol. 98, pp. 14-38, Jul. 2016. DOI: 10.1016/j.compedu.2016.03.010

[6] C. Castaño Garrido, U. Garay Ruiz, and S. Themistokleous, "De la revolución del software a la del hardware en educación superior," RIED. Revista Iberoamericana de Educación a Distancia, vol. 21, no. 1, p. 135, Sep. 2018. DOI: 10.5944/ried.21.1.18823

[7] J. M. T. C. José and M. Gómez, "Implementando scrum + rad para la gestión y desarrollo de proyectos de software en equipos de trabajo con personal limitado y eventual," vol. 8, pp. 52-64, 2016.

[8] J. Patricia and Z. Gamboa, "Evolución de las Metodologías y Modelos utilizados en el Desarrollo de Software. Evolution of the Methodologies and Models used in Software Development.," vol. 3, no. 10 , pp. $20-33,2018$.

[9] U. N. de C. Ludifisica, "PhysicsSensor Mobile Edition," Universidad Nacional de Colombia Sede Medellin, 2019. [Online]. Available: http://ciencias.medellin.unal.edu.co/ludifisica/. [Accessed: 28-Jan2019].

[10] PASCO, “Guía de Usuario Xplorer GLX 1,” 2018. 
[11] D. F. S. Dr. František Lustig, "e-laboratory project," Universidad de Charles en Praga República Checa, 2019. [Online]. Available: http://www.ises.info/index.php/en. [Accessed: 25-Jan-2019].

[12] R. Heradio, L. de la Torre, and S. Dormido, "Virtual and remote labs in control education: A survey," Annual Reviews in Control, vol. 42, pp. 1-10, Jan. 2016. DOI: 10.1016/j.arcontrol.2016.08.001

[13] D. Lowe, P. Newcombe, and B. Stumpers, "Evaluation of the Use of Remote Laboratories for Secondary School Science Education," Research in Science Education, vol. 43, no. 3, pp. 1197-1219, Jun. 2018. DOI: $10.1007 / \mathrm{s} 11165-012-9304-3$

[14] P. O. Javier García-Zubia, "WebLab-Deusto - Your labs, on the Internet," DeustoTech - Deusto Institute of Technology, vol. 1, 2019.

[15] Universidad de Medellín, Universidad Católica de Pereira, and Universidad Católica de Manizales, "Laboratorio Remoto y Virtual para la enseñanza de la Física, Physilab.” Medellin Colombia, p. 10, 2013

[16] University of Colorado Boulder, "Physics - PhET Simulations," University of Colorado Boulder, 2019. [Online]. Available: https://phet.colorado.edu/en/simulations/category/physics. [Accessed: 25-Jan-2019].

[17] D. F. Becerra-Rodríguez, C. Mora, A. F. Ordóñez Jiménez, and R. Sánchez Sánchez, "Enseñanza de la Ley de Ohm utilizando laboratorios virtuales con estudiantes de ingenierías de la Universidad Antonio Nariño," Am. J. Phys. Educ, vol. 10, no. 4, pp. 1-4, 2016.

[18] E. Figueiras, D. Olivieri, A. Paredes, and H. Michinel, "An open source virtual laboratory for the Schrödinger equation," European Journal of Physics, vol. 39, no. 5, p. 55802, 2018. DOI: 10.1088/13616404/aac999

[19] C. M. Enrique and G. E. Alzugaray, "Modelo de EnseñanzaAprendizaje para el Estudio de la Cinemática de un Volante Inercial usando Tecnologías de la Información y la Comunicación en un Laboratorio de Física," Formación universitaria, vol. 6, no. 1, pp. $3-$ 12, 2013.

[20] C. Infante Jiménez, "Propuesta pedagógica para el uso de laboratorios virtuales como actividad complementaria en las asignaturas teóricoprácticas," Revista Mexicana de Investigación Educativa RMIE, vol. 19, no. 62, pp. 917-937, 2014.

[21] G. Gunawan, A. Harjono, H. Sahidu, and L. Herayanti, "Virtual Laboratory of Electricity Concept to Improve Prospective Physics Teachers Creativity," Jurnal Pendidikan Fisika Indonesia, vol. 13, no. 2, pp. 102-111, Nov. 2017.

[22] Ö. K. Mirçik and A. Z. Saka, "Virtual Laboratory Applications in Physics Teaching," Canadian Journal of Physics, vol. 96, no. 7, p. cjp2017-0748, Jul. 2017.

[23] A. Á. Martínez and J. S. S. Díaz, "Internet de las cosas y herramientas de software libre aplicadas a la educación," Ingeniare, vol. 0, no. 22, pp. 11-18, 2018.

[24] J. G. Universidad de Murcia., A. M. Rivilla, and C. R. S. Juan, "Validación de requisitos funcionales de un Laboratorio Virtual Remoto como apoyo al blended learning," Revista de Educación a Distancia, vol. 0, no. 45, Oct. 2015.

[25] S. Saponara, P. Nuzzo, P. D'Abramo, and L. Fanucci, "Design methodologies and innovative architectures for mixed-signal embedded systems," Eurasip Journal on Embedded Systems, vol. 2010,2010

[26] M. A. Tosini et al., "Metodologías de Diseño para Sistemas Embebidos," XV WORKSHOP DE INVESTIGADORES EN CIENCIAS DE LA COMPUTACIÓN, pp. 713-717, 2013.

[27] J. G. F. Mendoza, J. A. A. España, J. B. M. Castro, J. G. D. Núñez, and E. N. Vaca, "Propuesta metodológica para validar la funcionalidad de software en sistemas embebidos," Pistas Educativas, vol. 38, no. 122, Dec. 2016.

[28] J. J. Morales Arias and C. J. Pardo Calvache, "Systematic review about the integration of model-driven software development and agile methodologies.," Informador Técnico. UPTC, vol. 80, no. 1, p. 87, Jan. 2016.

[29] J. R. Molina Ríos, M. P. Zea Ordóñez, M. J. Contento Segarra, and F. G. García Zerda, "Estado del arte: metodologías de desarrollo en aplicaciones web.," Revista Ciencia e Ingeniería., vol. 6, no. 3, p. 54, Sep. 2017.

[30] L. G. Palacio and G. U. Giraldo, "Modelo de requisitos para sistemas embebidos: Modelo de requisitos para sistemas embebidos," Revista Ingenierías Universidad de Medellín, vol. 7, no. 13, pp. 111-127, Jul. 2008 .
[31] M. R. Mollahoseini Ardakani, S. M. Hashemi, and M. Razzazi, "Adapting the scrum methodology for establishing the dynamic interorganizational collaboration," Journal of Organizational Change Management, vol. 31, no. 4, pp. 852-866, Jul. 2018.

[32] Incorporates IEEE, 1012-2016 Standard IEEE for System, Software, and Hardware Verification and Validation., IEEE. IEEE, 2017.

[33] T. I. Microchip Technology, "PIC12F675 Datasheet(PDF)," ALLDATASHEET.COM, 2019. [Online]. Available: http://www.alldatasheet.com/datasheetpdf/pdf/348702/MICROCHIP/PIC12F675.html. [Accessed: 29-Jan2019].

[34] Freescale semiconductor, "MMA8452q 12-bit/8-bit digital accelerometer," NXP Semiconductors Data sheet: Technical data, $2019 . \quad$ [Online]. Available: https://www.datasheetspdf.com/datasheet/search.php?sWord=mma84 52q. [Accessed: 29-Jan-2019].

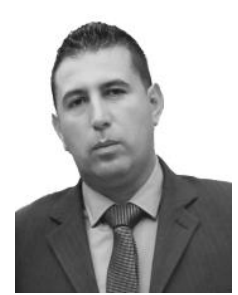

Jorge Enrique Chaparro-Mesa. Magister en Tecnología Informática. UPTC. Especialista en Redes de Alta Velocidad y Distribuidas, Universidad INCCA de Colombia; Ingeniero Electrónico, UPTC. Investigador Regional del OCyT para la elaboración del Plan Estratégico Departamental de Ciencia Tecnología e Innovación, PEDCTI de Casanare. Coordinador de Producción y Divulgación de la Investigación, Unitrópico, Coordinador (e) del Programa de Ingeniería de Sistemas, Director del Departamento de Tecnologías de la Información y las comunicaciones de Unitrópico, docente Investigador Tiempo Completo. Unitropico. Docente catedra UPTC. Delegado ante el CODECTI Casanare por Unitrópico, desde el año 2011 hasta el año 2016. Director cientifico Centro de desarrollo Tecnologico NetworkTIC.

ORCID: https://orcid.org/0000-0002-0752-5374

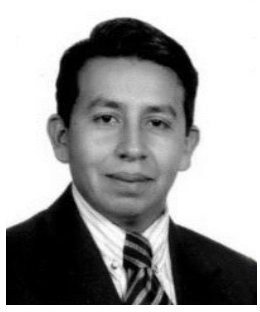

Nelson Barrera-Lombana. Doctor en ciencias de la Educación de la Uptc; Magister en Educación de la Uptc; Especialista en Soluciones Telemáticas de la Fundación Universitaria Autónoma de Colombia FUAC; Ingeniero Electrónico de la Uptc. Investigador y docente escalafonado de la Uptc; Coordinador del grupo de Investigación en Robótica y Automatización Industrial GIRA 2006-2011. Docente programa de ingeniería Electrónica tiempo completo de la Uptc 2002-2019; docente de tiempo completo Universidad Antonio Nariño UAN 2000-2002; director del programa de Ingeniería Electrónica de la Uptc 2019.

ORCID: https://orcid.org/0000-0002-3413-1709

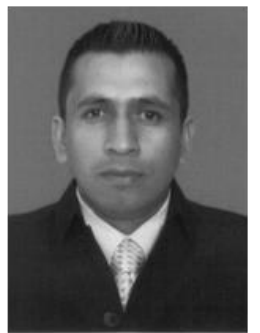

Fredy Alonso Leon-Socha. Ingeniero Electronico, UPTC. Especialista en desarrollo de aplicaciones moviles, SENA. Docente universitario, UPTC. Tutor virtual, AREANDINA. Director del Grupo de Investigacion NetworkTIC. Director de Desarrollo, innovación y nuevos productos, SASET SAS. Consultor TI. Autor de contenidos para cursos y capacitaciones en ambientes virtuales de aprendizaje.Asesor en diseño e implementación de proyectos tecnologicos en áreas de electrónica ,TIC y software.Análisis, diseño y ejecución de planes de marketing digital. Redactor de contenidos para plataformas virtuales.

ORCID: https://orcid.org/0000-0002-8310-8548 